\title{
Semiclassical theory of shot noise and its suppression in a conductor with deterministic scattering
}

\author{
C. W. J. Beenakker and II. van Houten \\ Philips Research Laboratories, 5600 JA Endhoven, The Netherlands
}

(Received 5 February 1991)

\begin{abstract}
A scattering theory for nonequilibrium current noise in a degenerate electron gas at finite temperature is developed, based on classical kinetic equations for the first two moments of the fluctuating distribution function $F(\mathbf{r}, \mathbf{p}, t)$. The result is a relation between the low-frequencynoise spectral density $P$ and the classical transmission-probability distribution function $T(\mathbf{r}, \mathbf{p})$. At zero temperature, $P=0$ if and only if $T$ takes on exclusively the values 0 and 1 . Shot noise is thus suppressed by (classical) determınıstıc scaltering in a degenerate electron gas.
\end{abstract}

The discreteness of the electron charge causes fluctuations in time of the electrical current flowing in response to a time-independent voltage difference. These fluctuations, which (unlike thermal fluctuations) persist down to zero temperature, are known as shot noisc. A recent theoretical development has been the derivation of a relation between the noise spectral density $P$ (per unit frequency bandwidth) and the transmission matrix $t$ of the conductor. ${ }^{1-5}$ The zero-frequency, zero-temperature shot-noise power of a two-terminal conductor (under conditions of a small voltage difference $V$ between the two terminals) was found to be given by ${ }^{4,5}$

$$
\begin{aligned}
P & =2 e|V| \frac{e^{2}}{h}-\operatorname{Tr} \underline{t} \underline{t}^{\dagger}\left(1-\underline{t} \underline{t}^{\dagger}\right) \\
& =2 e|V| \frac{e^{2}}{h} \sum_{n} T_{n}\left(1-T_{n}\right),
\end{aligned}
$$

in terms of the eigenvalues $T_{n}$ of the matrix product $\underline{t}^{\dagger}$ (cvaluated at the Fermi energy). This beautiful result tells us that shot noise is completely suppressed if all eigenvalues are either 0 or 1 . Experiments indicating a suppression of shot noise in a quantum point contact (i.e., a. constriction with a quantized conductance) have been reported by Li et al. ${ }^{\hat{\sigma}}$

In this paper we derive the semiclassical correspondence to Eq. (1), and to its finite-temperature form. ${ }^{4}$ The resulting expression is valid whenever the electron dynamics is governed by classical trajectories, in which case it represents a more convenient starting point for calculations than the fully quantum-mechanical formula. Suppression of shot noise is obtained from deterministic motion in a degenerate electron gas, which suggests that the phenomenon should be observable in a much larger class of conductors than the ballistic point contacts considered previously.

We apply the classical kinetic theory of nonequilibrium fluctuations to a scattering problem in a degenerate electron gas, in the spirit of Landauer's approach to electrical transport. ${ }^{7}$ The kinetic theory, due to Ganzevich, Gurevich, and Katilius, ${ }^{8}$ describes the development in time of the first two moments of the fluctuating distribution function $F(\mathbf{r}, \mathbf{p}, t)$. Both kinetic equations have the form of a Boltzmann equation. An alternative to this method of moments is to start from a kinetic equation for $F$ itself, in the form of a Boltzmann equation with a randomly fluctuating source term. ${ }^{9}$ That is the approach taken by Kulik and Omel'yanchuk, ${ }^{10}$ who first noted the suppression of shot noise in ballistic point contacts, but did not obtain the relation between noise and transmission probabilities of present interest.

The average $\langle F\rangle \equiv \bar{F}$ of $F$ (averaged over the timedependent fluctuations) satisfies the Boltzmann equation

$$
\left(\frac{d}{d t}+\mathcal{S}\right) \bar{F}(\mathbf{r}, \mathbf{p}, t)=0
$$

The derivative

$$
\frac{d}{d t} \equiv \frac{\partial}{\partial t}+\mathbf{v} \cdot \frac{\partial}{\partial \mathbf{r}}+\mathcal{F} \cdot \frac{\partial}{\partial \mathbf{p}}
$$

(with $\mathbf{v} \equiv \mathbf{p} / m$ ), accounts for the classical determın astic motion in the external (electromagnetic) force field $\mathcal{F}(\mathbf{r})$. The scattering term

$$
\mathcal{S} \bar{F}(\mathbf{r}, \mathbf{p}, t) \equiv \int d \mathbf{p}_{1} W\left(\mathbf{p}, \mathbf{p}_{1}\right)\left[\bar{F}(\mathbf{r}, \mathbf{p}, t)-\bar{F}\left(\mathbf{r}, \mathbf{p}_{1}, t\right)\right]
$$

describes the stochaslic effects of impurity scattering [with quantum-mechanical transition rate $W\left(\mathbf{p}, \mathbf{p}_{1}\right)$ between momenta $\mathbf{p}$ and $\mathbf{p}_{1}$ ]. We assume that the scattering is elastic, and disregard the electron-electron interaction. As discussed in Ref. 8, the correlation function $\left\langle\delta F(\mathbf{r}, \mathbf{p}, t) \delta F\left(\mathbf{r}^{\prime}, \mathbf{p}^{\prime}, t^{\prime}\right)\right\rangle$ (with $\delta F \equiv F-\bar{F}$ ) satisfies for $t>t^{\prime}$ the Boltzmann equation in the first set of variables, 


$$
\left(\frac{d}{d t}+\mathcal{S}\right)\left\langle\delta F(\mathbf{r}, \mathbf{p}, t) \delta F\left(\mathbf{r}^{\prime}, \mathbf{p}^{\prime}, t^{\prime}\right)\right\rangle=0 .
$$

The equal-time correlation is given by ${ }^{8}$

$$
\begin{aligned}
& \left\langle\delta F(\mathbf{r}, \mathbf{p}, t) \delta F\left(\mathbf{r}^{\prime}, \mathbf{p}^{\prime}, t\right)\right\rangle \\
& \quad=\delta\left(\mathbf{r}-\mathbf{r}^{\prime}\right) \delta\left(\mathbf{p}-\mathbf{p}^{\prime}\right) \bar{F}(\mathbf{r}, \mathbf{p}, t)\left[1-h^{d} \bar{F}(\mathbf{r}, \mathbf{p}, t)\right]
\end{aligned}
$$

( $d$ denotes the dimensionality of the conductor). The term containing Planck's constant must be retained in Eq. (6), since we are dealing with a degenerate electron gas (for which by definition $\vec{F} \sim h^{-d}$ ).

We now apply the kinetic theory to the two-terminal scattering geometry of Fig. 1. Two electron reservoirs, in equilibrium at chemical potentials $\mu_{1}$ and $\mu_{2}$, are connected by ideal leads to a conductor. We assume free motion in the leads $(\mathcal{F} \equiv 0 \equiv W$ in the leads), with specular scattering at their boundaries. The current $I(t)$ through a cross section $S_{1}$ of lead 1 is given by

$$
I(t)=e \int_{S_{1}} d \mathbf{y} \int d \mathbf{p} v_{x} F(\mathbf{r}, \mathbf{p}, t) .
$$

The $x$ coordinate is along the axis of lead 1 , and $\mathbf{y}$ is a vector perpendicular to the $x$ axis. The current has average $\bar{I}$ and fluctuations $\delta I(t) \equiv I(t)-\bar{I}$. We assume stationarity, i.e., a time independent $\bar{I}$ (and $\bar{F}$ ). The noise spectral density per unit frequency bandwidth, in the limit of zero frequency, is given by the time integral of the current-current correlation function,

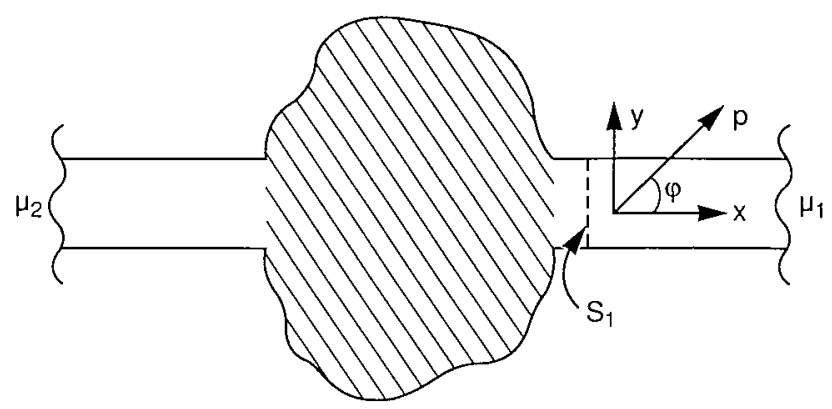

FIG. 1. Schematic drawing of a (disordered) conducting region (hatched), connected by ideal leads to two electron reservoirs (at chemical potentials $\mu_{1}$ and $\mu_{2}$ ). A cross section of lead 1 is indicated by $S_{1}$.

$$
\begin{aligned}
P & =4 \int_{0}^{\infty} d t\langle\delta I(t) \delta I(0)\rangle \\
& =4 e^{2} \int_{0}^{\infty} d t \int_{S_{1}} d \mathbf{y} \int_{S_{1}} d \mathbf{y}^{\prime} \int d \mathbf{p} \int d \mathbf{p}^{\prime} v_{x} v_{x}^{\prime} \chi
\end{aligned}
$$

where we have abbreviated $\chi \equiv\left\langle\delta F\left(\mathbf{r}^{\prime}, \mathbf{p}^{\prime}, t\right) \delta F(\mathbf{r}, \mathbf{p}, 0)\right\rangle$. We need to determine $\chi$ for $t>0$, and for points $\mathrm{r}^{\prime}=$ $\left(x, \mathbf{y}^{\prime}\right)$ and $\mathbf{r}=(x, \mathbf{y})$ on $S_{1}$. It is convenient to consider separately the incoming states $\left(p_{x}>0\right)$ and the outgoing states $\left(p_{x}<0\right)$.

Electrons with $p_{x}>0$ reach reservoir 1 without further crossing of $S_{1}$. Thus, $\chi$ contains only a term proportional to $\delta(t)$

$\chi\left(p_{x}>0\right)=\delta\left(\mathbf{r}^{\prime}-\mathbf{r}-\mathbf{v}^{\prime} t\right) \delta\left(\mathbf{p}^{\prime}-\mathbf{p}\right) \bar{F}(\mathbf{r}, \mathbf{p})\left[1-h^{d} \bar{F}(\mathbf{r}, \mathbf{p})\right]=\frac{1}{\left|v_{x}^{\prime}\right|} \delta(t) \delta\left(\mathbf{y}^{\prime}-\mathbf{y}\right) \delta\left(\mathbf{p}^{\prime}-\mathbf{p}\right) \bar{F}(\mathbf{r}, \mathbf{p})\left[1-h^{d} \bar{F}(\mathbf{r}, \mathbf{p})\right]$.

Electrons with $p_{x}<0$ can be reflected back from the conductor into the reservoir through $S_{1}$. Let $R_{n}$ denote the probability that the clectron with initial coordinates $x, \mathbf{y}$ on $S_{1}$, and initial momentum $\mathbf{p}$, returns after a time $t_{n}$ to a point $x, \mathbf{y}_{n}$ on $S_{1}$, with final momentum $\mathrm{p}_{n}$. The set of possible return paths is denoted symbolically by $\{n\}$. The total reflection probability $R(\mathbf{r}, \mathbf{p})$ for an electron starting from $\mathrm{r}, \mathrm{p}$ on $S_{1}$ equals $\sum_{\{n\}} R_{n}$. The correlation function $\chi$ for $p_{x}<0$ contains terms proportional to $R_{n} \delta\left(t-t_{n}\right)$, in addition to a $\delta(t)$ term as in Eq. (9):

$$
\chi\left(p_{x}<0\right)=\frac{1}{\left|v_{x}^{\prime}\right|}\left(\delta(t) \delta\left(\mathbf{y}^{\prime}-\mathbf{y}\right) \delta\left(\mathbf{p}^{\prime}-\mathbf{p}\right)+\sum_{\{n\}} R_{n} \delta\left(t-\iota_{n}\right) \delta\left(\mathbf{y}^{\prime}-\mathbf{y}_{n}\right) \delta\left(\mathbf{p}^{\prime}-\mathbf{p}_{n}\right)\right) \bar{F}(\mathbf{r}, \mathbf{p})\left[1-h^{d} \bar{F}(\mathbf{r}, \mathbf{p})\right]
$$

The average distribution function $\bar{F}$ in the lead can bc expressed in terms of the average distribution functions $\bar{F}_{\alpha}^{\prime}$ in the reservoirs $(\alpha=1,2)$. Since the reservoirs are in equilibrium, $\bar{F}_{\alpha}$ is the Fermi-Dirac distribution in phase space,

$$
\bar{F}_{\alpha}(\mathrm{r}, \mathrm{p})=h^{-d} f\left(E-\mu_{\alpha}\right),
$$

where $f(x)=[1+\exp (x / k T)]^{-1}$. The energy $E$ of the electron is a constant of the motion in the conduc- tor, where all scattering is assumed to be elastic (inelastic scattering in this formalism occurs only in the reservoirs ${ }^{7}$ ). All electrons in lead 1 with $p_{x}<0$ originate from reservoir 1 , so that

$$
\bar{F}(\mathbf{r}, \mathbf{p})=h^{-d} f\left(E-\mu_{1}\right), \text { if } p_{x}<0 .
$$

An electron at $\mathbf{r}, \mathbf{p}$ in lead 1 with $p_{x}>0$ may have originated either from reservoir 2 , with transmission probability $T(\mathbf{r}, \mathbf{p})$, or from reservoir 1 , with probability $1-T(\mathbf{r}, \mathbf{p})$. One thus has 


$$
\vec{F}(\mathbf{r}, \mathbf{p})=h^{-d} f\left(E-\mu_{2}\right) T(\mathbf{r}, \mathbf{p})+h^{-d} f\left(E-\mu_{1}\right)[1-T(\mathbf{r}, \mathbf{p})] \text {, if } p_{x}>0 .
$$

On substitution of Eqs. (9)-(13) into Eq. (8), and carrying out the integrations over $t, \mathbf{y}^{\prime}$, and $\mathbf{p}^{\prime}$, we obtain the result

$$
\begin{gathered}
P=4 e^{2} \int_{S_{1}} d \mathbf{y} \int d \mathbf{p}\left|v_{x}\right| h^{-d}\left\{\Theta\left(p_{x}\right) \frac{1}{2}\left[f_{2} T+f_{1}(1-T)\right]\left[1-f_{2} T-f_{1}(1-T)\right]+\Theta\left(-p_{x}\right)\left(\frac{1}{2}-R\right) f_{1}\left(1-f_{1}\right)\right\} \\
=4 \frac{e^{2}}{h} \int_{0}^{\infty} d E(2 E / m)^{1 / 2} h \rho \int_{S_{1}} d \mathbf{y} \int_{-\pi / 2}^{\pi / 2} \frac{d \phi}{2 \pi} \cos \phi\left\{\frac{1}{2}\left[f_{2} T+f_{1}(1-T)\right]\right. \\
\left.\times\left[1-f_{2} T-f_{1}(1-T)\right]-\left(\frac{1}{2}-T\right) f_{1}\left(1-f_{1}\right)\right\}
\end{gathered}
$$

[abbreviating $f_{\alpha} \equiv f\left(E-\mu_{\alpha}\right)$ ]. In the second equality we have eliminated $R$ in favor of $1-T$ by using current conservation. The unit-step function is denoted by $\Theta, \rho$ is the density of states $\left(\rho=m / 2 \pi \hbar^{2}\right.$ in two dimensions, in the absence of spin degeneracy), and $\phi$ the angle of $p$ with the $x$ axis. The angular average of $\mathrm{p}$ is written down for the case $d=2$, and should be replaced by a spherical angular average in the case of a three-dimensional conductor.

To write Eq. (14) in a more transparent way, we define the average $\langle\cdots\rangle_{E}$, over $\mathbf{y}$ and $\phi$ at energy $E$, by

$\langle T(\mathbf{r}, \mathbf{p})\rangle_{E} \equiv(2 E / m)^{1 / 2} h \rho \int_{S_{1}} d \mathbf{y} \int_{-\pi / 2}^{\pi / 2} \frac{d \phi}{2 \pi} \cos \phi T(\mathbf{r}, \mathbf{p})$.

With this notation, and after a rearrangement of terms, Eq. (14) takes the form

$$
\begin{aligned}
& P=P_{1}+P_{2}, \\
& P_{1}=2 \frac{e^{2}}{h} \int_{0}^{\infty} d E\langle T(\mathbf{r}, \mathbf{p})\rangle_{E}\left[f_{1}\left(1-f_{1}\right)+f_{2}\left(1-f_{2}\right)\right], \\
& P_{2}=2 \frac{e^{2}}{h} \int_{0}^{\infty} d E\langle T(\mathbf{r}, \mathbf{p})[1-T(\mathbf{r}, \mathbf{p})]\rangle_{E}\left(f_{1}-f_{2}\right)^{2} .
\end{aligned}
$$

This is the required classical correspondence to the results of Lesovik ${ }^{4}$ and Büttiker. ${ }^{5}$ Let us examine scvcral limiting forms of Eq. (16).

In equilibrium, $\mu_{1}=\mu_{2}=E_{\mathrm{F}}$, so that $f_{1}=f_{2}=$ $f\left(E-E_{\mathrm{F}}\right)$. Hence, $P_{2}=0$, while $P_{1}=P_{\text {thermal }}$ is given by

$$
P_{\text {thermal }}=4 \frac{e^{2}}{h} \int_{0}^{\infty} d E\langle T(\mathbf{r}, \mathbf{p})\rangle_{E} f(1-f)
$$

By averaging Eq. (7), and using Eqs. (12) and (13), one readily obtains the linear-response conductance

$$
G=-\frac{e^{2}}{h} \int_{0}^{\infty} d E\langle T(\mathbf{r}, \mathbf{p})\rangle_{E} \frac{d f}{d E} .
$$

In view of the identity $f(1-f)=-k T f^{\prime}$, Eqs. (17) and (18) are in accord with the Nyquist formula $P_{\text {thermal }}=$
$4 k T G$ for the thermal noise in equilibrium. ${ }^{2}$

At zero temperature, $f_{\alpha}=\Theta\left(\mu_{\alpha}-E\right)$. Hence, $P_{1}=0$, while $P_{2}=P_{\text {shot }}$ is given by

$$
P_{\text {shot }}=2 e|V| \frac{e^{2}}{h}\langle T(\mathbf{r}, \mathbf{p})[1-T(\mathbf{r}, \mathbf{p})]\rangle_{E_{\mathrm{F}}},
$$

to linear order in $\mathrm{eV}=\mu_{1}-\mu_{2}$. Classical determmistic transport corresponds to $\mathcal{S}=0$ in the Boltzmann equations (2) and (5). In that case, the transmission probability $T(\mathbf{r}, \mathbf{p})$ takes on exclusively the values 0 and 1 , so that $P_{\text {shot }}=0$. Conductance quantization is thus not required for the suppression of shot noise, nor is the absence of backscattering a requirement. In the opposite stochastic regime of diffusive transport, $T(\mathbf{r}, \mathbf{p}) \ll 1$, so that $P_{\text {shot }}=2 e|V| G=2 e|\bar{I}|$ equals the noise power of a Poisson process ("full shot noise"). Deterministic motion between two reservoirs at zero temperature violates the assumption of uncorrelated current pulses on which the Poisson process is based, so that the noise remains below the full shot-noise level.

At nonzero temperature and nonzero applied voltage, the noise power crosses over from the thermal form (17) to the shot-noise form (19) when $k T \sim e V$. This crossover is described by the formula

$$
\begin{aligned}
P=\frac{e^{2}}{h}[ & 4 k T\langle T(\mathbf{r}, \mathbf{p}) T(\mathbf{r}, \mathbf{p})\rangle_{E_{\mathrm{F}}} \\
& \left.+2 e V \operatorname{coth}\left(\frac{e V}{2 k T}\right)\langle T(\mathbf{r}, \mathbf{p})[1-T(\mathbf{r}, \mathbf{p})]\rangle_{E_{\mathrm{F}}}\right]
\end{aligned}
$$

which follows from Eq. (16) by evaluating the energy integral (for $k T, e V \ll E_{\mathrm{F}}$, and assuming that $\langle T\rangle_{E}$ and $\left\langle T^{2}\right\rangle_{E}$ are approximately $E$ independent in the intervals $k T$ and $\mathrm{eV}$ around $E_{\mathrm{F}}$ ) ${ }^{11}$ Equation (20) describes the effect of thermal averaging on the shot-noise power. A nonzero temperature has another, indirect, effect on shot noise through inelastic-scattering in the conductor (neglected in this, and other, ${ }^{1-5}$ scattering theories). A finite inelastic-scattering length $l_{\mathrm{nn}}$, much smaller than the length $L$ of the conductor, decreases $P_{\text {shot }}$ by a factor of $L / l_{\mathrm{m}}$ (being the number of uncorrelated segments in the 
conductor), while $P_{\text {thermal }}$ remains essentially unaffected. It would be of interest to study the effect on the noise of inelastic scattering from additional (zero-current drawing) reservoirs connected to the conductor. This could be done by using the multiterminal formulas of Ref. 5 .

Comparison of Eq. (19) with Eq. (1) implies thè classical correspondences

$$
\begin{aligned}
& \lim _{h \rightarrow 0} \operatorname{Tr} \underline{t} \underline{t}^{\dagger}(E)=\langle T(\mathbf{r}, \mathbf{p})\rangle_{E} \\
& \lim _{h \rightarrow 0} \operatorname{Tr} \underline{t}^{\dagger} \underline{t}^{\dagger}(E)=\langle T(\mathbf{r}, \mathbf{p}) T(\mathbf{r}, \mathbf{p})\rangle_{E} .
\end{aligned}
$$

The limit (21) follows also from a comparison of Eq. (18) (at zero temperature) with the Landauer formula $G=\left(e^{2} / h\right) \operatorname{Tr} \underline{t} \underline{t}^{\dagger}$. A direct proof of the limit $(22)$ is complicated by the fact that the off-diagonal components of the matrix $\left(\underline{t} \underline{t}^{\dagger}\right)_{n m}=\sum_{p} t_{n p} t_{m p}^{*}$ do not vanish as $h \rightarrow 0$, but nonetheless have no obvious classical interpretation. [These off-diagonal components do not appear in Eq. (21).] We surmise that a transformation from a basis of transverse modes (which has for cach mode an $h$-independent uncertainty $\Delta y$ in the $y$ coordinate), to a wave-packet basis (for which both $\Delta y \rightarrow 0$ and $\Delta p_{y} \rightarrow 0$ as $h \rightarrow 0$ ), will diagonalize the matrix $\underline{t} \underline{t}^{\dagger}$ in the limit $h \rightarrow 0$, but we do not have a satisfactory proof of this.

In summary, we have presented a semiclassical scattering theory of current noise, which relates the noise power $P$ to the transmission probability distribution function $T(\mathbf{r}, \mathbf{p})$. The zero-temperature equivalence $P=$ $0 \Leftrightarrow T(\mathbf{r}, \mathbf{p})=0,1$ for all states at the Fermi energy, provides the classical correspondence to the quantummechanical criterion ${ }^{4,5} T_{n}=0,1$ for the suppression of shot noise, and identifies deterministic scattering as the semi-classical origin of this effect. An experimental demonstration of this theoretical result could be obtained from noise measurements in a high-mobility twodimensional electron gas, in which macroscopic scatterers have been introduced artificially by selective etching or by gate electrodes. The scattering potential can then be made to vary slowly on the scale of a wavelength, so that classical deterministic scattering prevails. Suppression of the low-temperature shot noise is predicted, even if the potential causes strong backscattering.

We acknowledge the stimulating support of M. F. II. Schuurmans.
${ }^{1}$ V. A. Khlus, Zh. Eksp. T'oor. Fiz. 93, 2179 (1987) [Sov. Phys. - JETP 66, 1243 (1987)]. In this paper, which has not been noticed in more recent work, an expression of the form (1) is derived by a semiclassical Green's-function method.

${ }^{2}$ R. Landauer, Physica D 38, 226 (1989).

${ }^{3}$ B. Yurke and G. P. Kochanski, Phys. Rev. B 41, 8184 (1990).

${ }^{4}$ G. B. Lesovik, Pis'ma Zh. Eksp. Teor. Fiz. 49, 513 (1989) [JETP Lett. 49, 592 (1989)].

${ }^{5}$ M. Büttiker, Phys. Rev. Lett. 65, 2901 (1990).

${ }^{6}$ Y. P. Li, D. C. Tsui, J. J. IJeremans, J. $\Lambda$. Simmons, and G. W. Weimann, Appl. Pliys. Lett. 57, 774 (1990). Noise properties of quantum point contacts have also been investigated by G. Timp, R. E. Behringer, and J. E. Cunningham, Pliys. Rev. B 42, 9259 (1990); C. Dekker, A. J. Scholten, F. Liefrink, R. Eppenga, H. van Houten, and C. T. Foxon (unpublished). The two latter papers study the noise spectral density due to resistance fluctuations, which depends quadratically on the applied voltage (in contrast to the linear $V$-dependence characteristic of shot noise at small $V$ ).
${ }^{7}$ R. Landauer, IBM J. Res. Dev. 1, 223 (1957); 32, 306 (1988).

${ }^{8}$ S. V. Gantsevich, V. L. Gurevich, and R. Katilius, Rivista Nuovo Cimento 2 (5), (1979). For a summary, see E. M. Lifshitz and L. P. Pitaevskii, Physical Kinetics (Pergamon, Oxford, 1981), paragraph 20.

${ }^{9}$ B. B. Kadomtsev, Zh. Eksp. Teor. Fiz. 32, 943 (1957) [Sov. Phys. - JETP 5, 771 (1957)]; Sh. M. Kogan and A. Ya. Shul'man, ibid. 56, 862 (1969) ibid. 29, 467 (1969)].

${ }^{10}$ I. O. Kulik and A. N. Omel'yanchuk, Fiz. Nisk. Temp. 10, 305 (1984) [Sov. J. Low Temp. Phys. 10, 158 (1984)].

${ }^{11}$ Equation (20) cannot be used to describe the shot noise in a vacuum tube with a thermionic emitter, since there the transmission probability varies strongly over $k T$. Instead, we can simplify Eq. (16) by omitting all terms quadratic in $f$ (since $f \ll 1$ for the energies where $\langle T\rangle_{E} \neq 0$ ). In the saturation regime, $f_{2}(E)=0$ when $\langle T\rangle_{E} \neq 0$ (the indices 1,2 label cathode and anode). One then obtains from Eq. (16): $P=2\left(e^{2} / h\right) \int d E\langle T\rangle_{E} f_{1}(E)=2 e \bar{I}$, which is the full shotnoise level, regardless of whether $T$ is deterministic or not. It is only in the low-temperature limit that a deterministic $T$ results in the suppression of shot noise. 